\title{
Quality of Management of Severe Malaria Cases in Children under Five: A Case of Four Health Facilities in the Littoral Department in Benin
}

\author{
Gloria Ayivi-Vinz ${ }^{*}$, Ghislain E. Sopoh1 ${ }^{1}$, Charles Sossa1, Chabi Olaniran Alphonse Biaou1, \\ Abdou-Rahim Ouro-Koura², Michel Makoutodé1, Edgar-Marius Ouendo' ${ }^{1}$ \\ ${ }^{1}$ Institut Régional de Santé Publique Comlan Alfred Quenum, Université d'Abomey, Calavi, Bénin \\ ${ }^{2}$ Centre de Formation en Santé Publique (CFSP), Lomé, Togo \\ Email: *ayivivinzgloria@gmail.com
}

How to cite this paper: Ayivi-Vinz, G., Sopoh, G.E., Sossa, C., Biaou, C.O.A., Ouro-Koura, A.-R., Makoutodé, M. and Ouendo, E.-M. (2020) Quality of Management of Severe Malaria Cases in Children under Five: A Case of Four Health Facilities in the Littoral Department in Benin. Advances in Infectious Diseases, 10, 163-175. https://doi.org/10.4236/aid.2020.104014

Received: May 29, 2020

Accepted: July 28, 2020

Published: July 31, 2020

Copyright $\odot 2020$ by author(s) and Scientific Research Publishing Inc. This work is licensed under the Creative Commons Attribution-NonCommercial International License (CC BY-NC 4.0). http://creativecommons.org/licenses/by-nc/4.0/ (c) (i) (9) Open Access

\begin{abstract}
Background: Benin republic is a sub-Saharan African country endemic to malaria caused by Plasmodium falciparum. To reduce the death rate due to this scourge, it is essential to ensure quality care, especially for children under five years who are the most vulnerable. The main objective of this study is to assess the quality of severe malaria case management in patients under five years. Methods: Descriptive and analytical cross-sectional study was performed in health facilities in Cotonou city, the economic capital of Benin Republic. Study sample included 205 suspected cases of severe malaria among children aged under five years, in four health facilities. In each of hospitals, all children under five years with severe malaria presumption were enrolled in the study. Quality was assessed through five components: diagnosis, treatment, patient monitoring, counselling/advice, and patient's follow-up. Based on the Benin Republic's malaria case management standard protocol, the assessment criteria of each component were defined. Results: The sex ratio was 1.25 boys to a girl. The median of hospitalisation duration was 3 days with first quartile $(\mathrm{Q} 1)=2$ and third quartile $(\mathrm{Q} 3)=5$. Among 205 severe malaria cases included, 157 (76.59\%) were not managed in accordance with the requirements of the national protocol of malaria case management. The different components of cases management are not all implemented during the care of patients. Conclusion: There is a need for training to health workers on implementation of severe malaria in children under 5 years protocol. Also, there is a need for investigation about factors associated with malaria protocol implementation.
\end{abstract}

\section{Keywords}

Severe Malaria, Case Management, Protocol Observance, Quality of Care, 
Benin

\section{Background}

Benin Republic is a sub-Saharan African country endemic to malaria caused by Plasmodium falciparum. In Benin, malaria affects $15.5 \%$ of the general population [1]. The Malaria Indicators Surveys (MIS) of 2015 reports a parasite prevalence of $38.8 \%$ (confidence interval (CI) 35.7 - 41.9) in children under five years of age [2]. To reduce mortality caused by malaria, it is essential to ensure quality care, especially for children under five years who are the most vulnerable.

Over the past decades, Benin National Malaria Control Program (NMCP) has intensified interventions for malaria control. The adoption of Artemisinin-based Combinations Therapies (ACTs); leading mass distribution and awareness campaigns on the use of Long-Lasting Insecticidal Nets (LLINs); the adoption of Intermittent Preventive Treatment with Sulfadoxine/Pyrimethamine (IPT-sp) for the prevention of malaria in pregnant women; the presidential initiative of free malaria care for children under five and pregnant women, are the key elements. Also, adherence to the global initiative "Roll Back Malaria", increased funding for malaria control and the implementation of the Health System Performance Enhancement Project (PRPSS) helped strengthen malaria control interventions [3] [4] [5]. In addition, the establishment of an integrated supervision system to improve health care provider performance is one of the critical elements of the performance monitoring mechanism of the National Malaria Control Strategic Plan [4]. Thus, the NMCP strengthened the skills of health workers in the public sector through the training on the protocol for the management of malaria case at all levels of the health pyramid.

As far as severe malaria is concerned, the malaria case management protocol recommends that treatment should be done in only public and private reference health facilities such as private polyclinics, private hospitals, district hospitals, departmental hospitals or national hospitals [6]. The presumptive diagnosis of severe malaria is based on the identification of one of the signs of severity. Those signs include the inability to drink or suckle, uncontrollable vomiting, convulsions, lethargy or unconsciousness, disturbances of consciousness (coma), severe anaemia or severe pallor, prostration, breathing difficulties, haemoglobinuria, jaundice and abnormal bleeding. Confirmation of the diagnosis of malaria by microscopy or Rapid Diagnostic Test (RDT) is mandatory regardless of age. The protocol indicates that artemisinin derivatives or quinine be administered according to defined dosages to treat severe malaria cases. For artemisinin derivatives, the first three parenteral dosages are mandatory regardless of the patient's condition. As soon as possible, the relay is done with Artemisinin-based Therapeutic Combinations, orally to complete the seven days of treatment. The recommended dose of Artesunate is $2.4 \mathrm{mg} / \mathrm{kg}$, in three time (first, after twelve and twenty-four hours) slow intravenous injection. The following days, the treat- 
ment is administered once a day at the same dose $(2.4 \mathrm{mg} / \mathrm{kg})$. The recommended dose of Artemether is $3.2 \mathrm{mg} / \mathrm{kg}$, in intramuscular injection on the first day, then $1.6 \mathrm{mg} / \mathrm{kg}$ daily from the following day. Quinine is used intravenously in $10 \%$ glucose serum at $10 \mathrm{mg} / \mathrm{kg}$ every eight hours. As soon as the treatment is initiated parenterally, it is recommended that the three infusions of quinine be completed within the first 24 hours. The daily dose should not exceed $1800 \mathrm{mg}$ of quinine salts even if the patient weighs more than $60 \mathrm{~kg}$. Similarly, the relay is taken orally as soon as possible, and the total duration of treatment is seven days.

The guidelines explain that rigorous monitoring of the vital signs (temperature, pulse, respiratory rate and blood pressure two times per day), the state of consciousness, diuresis of the 24 hours and aspect of the urine, are necessary. As to the patient monitoring, the protocol recommends a daily visit by a health professional during hospitalisation. In addition, the protocol recommends that advice is given to the patient to continue treatment with adherence to the correct dosage and feeding, return to follow-up appointments and observe the preventive measures of malaria. After discharge, it is recommended follow-up appointments the patient on fourteenth and twenty-eighth days and every three months if there are persistent sequelae (paralysis, blindness, deafness, ataxia, epilepsy or psychomotor retardation) until the disappearance of the sequelae. Through those case management requirements, NMCP's main goals are to avoid death, and prevent the occurrence of sequelae, especially in children.

Despite multiple intervention's effects, the burden of malaria persists in Benin [1] [2]. According to health statistics of 2015, malaria is one of the primary reasons for consultation and hospitalisation in health facilities in Benin. It also accounts for $42.9 \%$ in hospitalised patients under five years of age [1]. The national incidence of severe malaria, according to the NMCP is $1.7 \%$ in 2015. For children under five years, this indicator is even higher. Benin's 2015 Health statistics report the incidence of severe malaria at $5.3 \%$ in 0 - 11 months patients and $5.2 \%$ in 12 - 59 months patients [1]. Severe malaria is the leading cause of death (28\%) for all patients. Among children under five years especially, $38 \%$ of deaths are due to severe malaria [1].

To reduce malaria-related mortality, it is essential to maintain the efforts made for the primary prevention of malaria but also to ensure quality care for patients suffering from severe malaria, especially children under five years who are the most vulnerable. For this purpose, there is a need of evidence on the quality of the management of severe malaria in health facilities. This study assesses the quality of severe malaria cases management in both private and public health facilities in the south Benin Republic.

\section{Methods}

This is a descriptive and analytical cross-sectional study and data were collected in Cotonou from June to July 2017. Data collection was done at the paediatric, emergency, or general medical services of targeted hospitals. Four hospitals were 
selected: "Suru Léré" hospital, "Ménontin" hospital, "Saint Luc" hospital, a "Saint Jean" Medical center. The choice of these health facilities was based on the level of reference in health pyramid. One this paper, good quality is defined as the management of cases of severe malaria in accordance with the national protocol for the management of malaria cases in Benin. This protocol is inspired by the WHO recommendations for malaria cases management [6] [7]. Quality was assessed using the following components: diagnosis, treatment, patient monitoring, counselling/advice, and patient follow-up [6].

\subsection{Study Population}

This study adopted census technique. Children under five years are hospitalised (stay in a health facility for more than 24 hours) with severe malaria presumption in each of the targeted hospitals were eligible for enrolment in the study. Specifically, patients aged from 0 to 59 months who completed treatment in one of the targeted hospitals for severe malaria as recorded in their folder and/or presenting at least one of the signs of gravity listed above with consent (child's parent agreement) to participate in the study. Patients who have spent less than 24 hours in a hospital, and/or whose parent or guardian (i.e. the main respondent) refused to participate in the study were not included. Also excluded are patients who are still on admission at the end of the data collection period and those without consent to participate. In total, the study enrolled two hundred and twenty (220) children under five years with severe malaria in the four targeted hospitals during the data collection period. Among these cases, 205 children are included in our study sample after meeting the inclusion criteria (Figure 1).

\subsection{Measuring and Assessing the Quality of Severe Malaria Case Management}

Using Benin Republic's malaria case management protocol, the stages of severe malaria care management process were identified. All these stages constitute the components of quality assessment. For each of this stage, the protocol explains what must be practiced. Table 1 summarizes elements of measurement of each component and assessment criterion.

\subsection{Data Processing and Analysis}

Data were processed and analysed using the Stata version 11 software. Associations between different components were explored through a chi-squared test or Fisher's exact test (if relevant) with the level of statistical significance $\alpha=5 \%$. A Cronbach test was performed to demonstrate that the five components used to assess the quality of cases management were distinct or related.

\section{Results}

The sex ratio was 1.25 boys to a girl. The median duration of hospitalisation was 


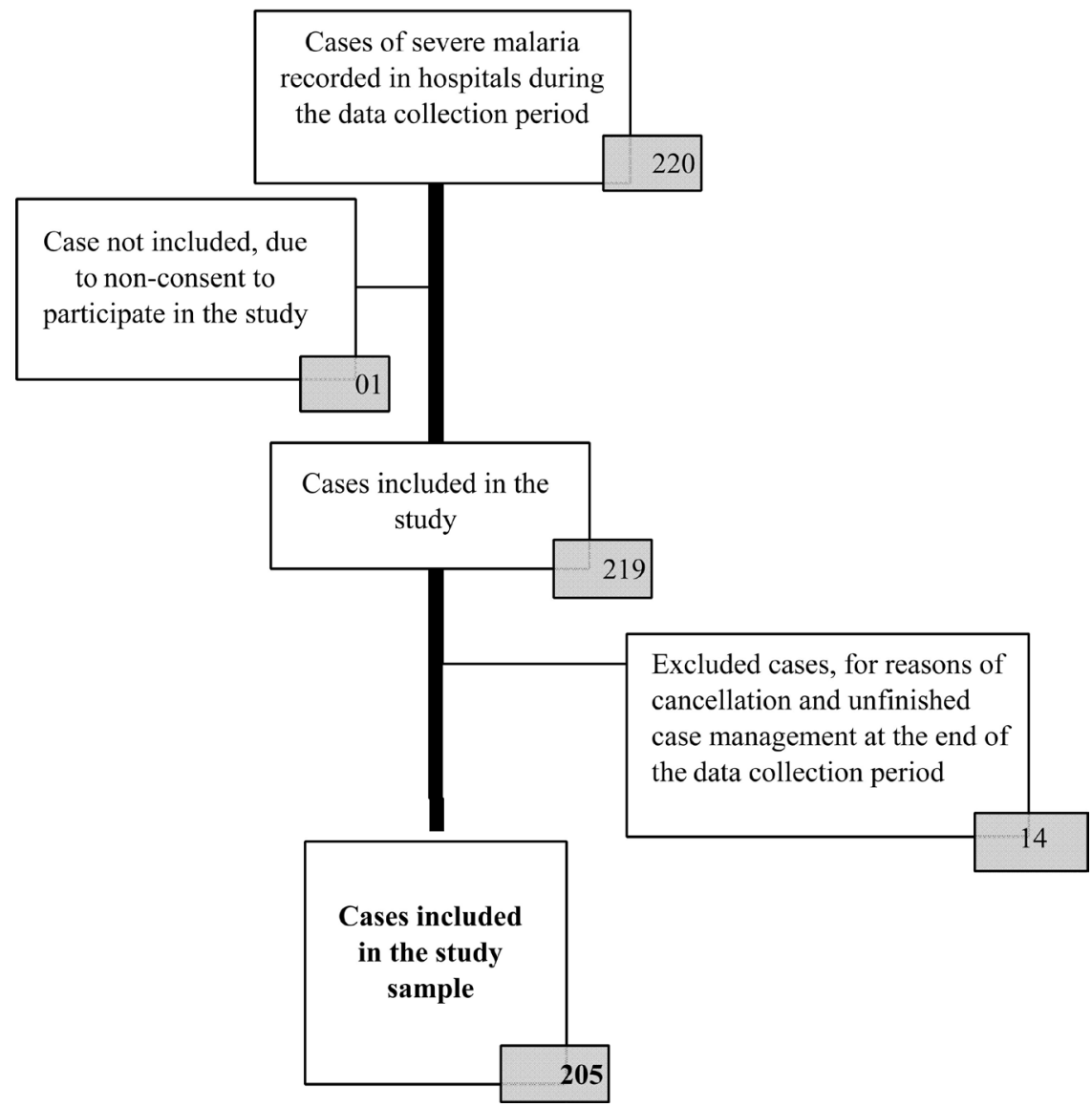

Figure 1. Flow diagram of severe malaria cases recorded in children under 5 years supported in four health facilities in Cotonou in June-July 2017.

Table 1. Sub-items of the assessment criteria of the components of management of malaria severe cases.

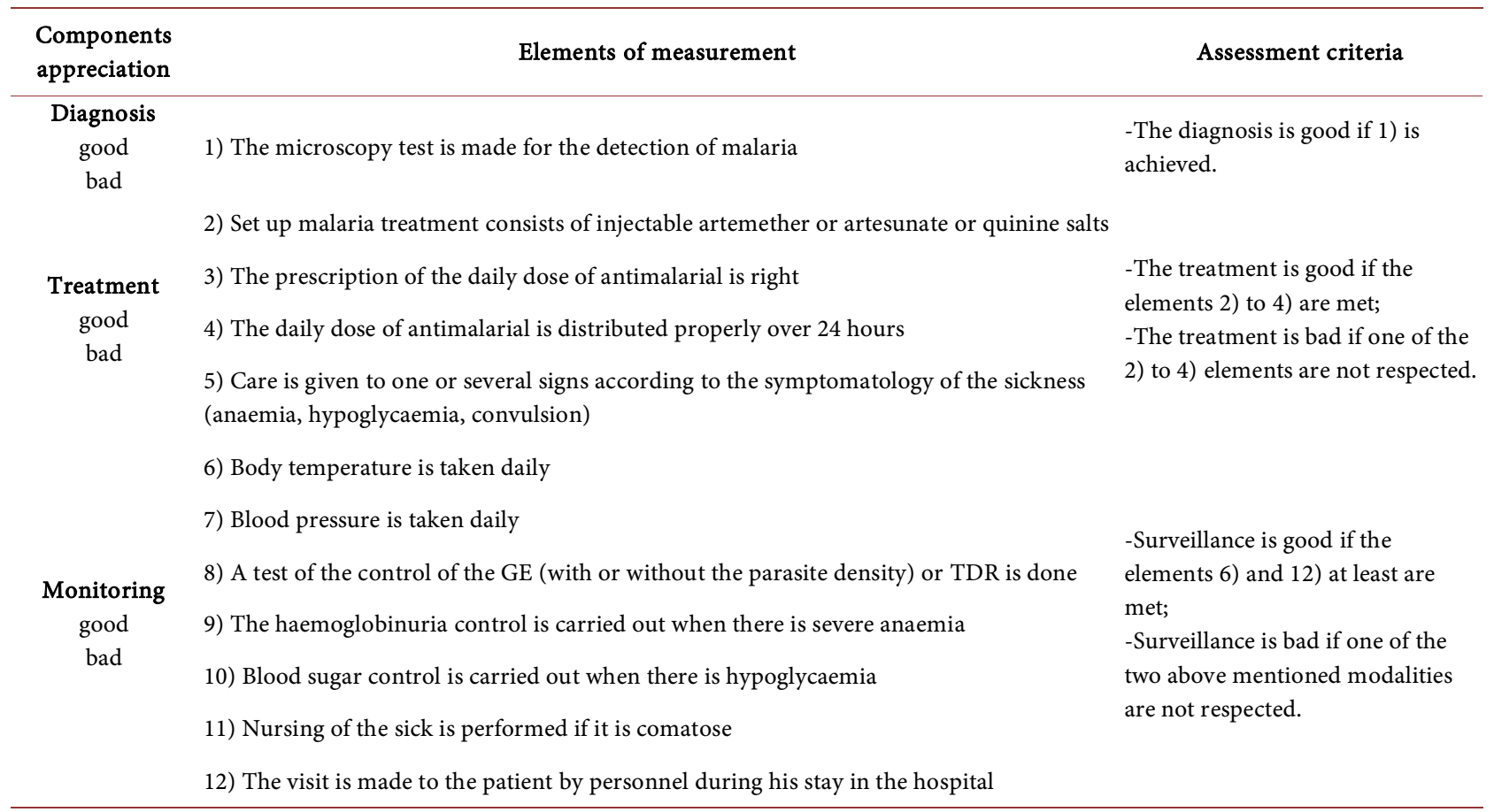




\section{Continued}

\begin{tabular}{|c|c|c|}
\hline Counselling & 13) The patient or his respondent received information about his illness & $\begin{array}{l}\text {-The council is sufficient if the } \\
\text { elements 13), 14) and 15) are met; }\end{array}$ \\
\hline $\begin{array}{c}\text { sufficient } \\
\text { not enough }\end{array}$ & $\begin{array}{l}\text { 14) The patient or his respondent received information on compliance with his treatment } \\
\text { 15) The patient or his respondent received information about the prevention of malaria }\end{array}$ & $\begin{array}{l}\text {-The council is not enough if one } \\
\text { or more of the three elements are } \\
\text { not met. }\end{array}$ \\
\hline $\begin{array}{l}\text { Follow-up } \\
\text { good } \\
\text { bad }\end{array}$ & $\begin{array}{l}\text { 16) The rest of the antimalarial treatment is prescribed to the patient out of the hospital } \\
\text { 17) An appointment is given to the patient for follow-up after his release from the } \\
\text { hospital }\end{array}$ & $\begin{array}{l}\text {-Follow-up is good if one of the } \\
\text { elements 16) and 17) is respected; } \\
\text {-Follow-up is bad if neither of the } \\
\text { two methods above is met. }\end{array}$ \\
\hline
\end{tabular}

3 days with first quartile $(\mathrm{Q} 1)=2$ and third quartile $(\mathrm{Q} 3)=5$. The median age of children included in our study was 30 months $[\mathrm{Q} 1=18$; Q3 = 40]. Thirty children (14.63\%) aged less one year old and 175 (85.37\%) aged 1 to 5 years. The most record complication was "severe malaria anaemia" (122 or 59.51\%). Figure 2 shows the classification of the clinical status of children.

\subsection{Diagnosis}

A confirmatory biologic test was performed in 184 out of the 205 cases (89.76\%). A total of 21 cases $(10.24 \%)$ were treated directly by a presumptive diagnosis. The diagnosis was confirmed positive by the microscopy for 159 children (86.41\%) and by RDT for 12 children (6.52\%). For the remaining 13 children, the outcome of the microscopy was negative (7.07\%) even though they received treatment for malaria.

\subsection{Treatment}

Two hundred and four among the 205 of children (99.51\%) in care received treatment for malaria. These cases were treated with quinine salt (17 or $8.33 \%$ ), artesunate (179 or $87.75 \%$ ) and artemether (8 or $3.92 \%)$. Antimalarial treatment protocols (dosage and delay) were respected in 135 out of the 204 cases. Figure 3 summaries diagnosis and treatment of severe malaria cases.

\subsection{Monitoring Advice and Follow-Up of Patients}

Control test of microscopy or "RDT" control test was prescribed for $49.27 \%$ of patients (101 out of 205 patients). For the 198 hospitalised patients, 196 (i.e. 98.99\%) received at least one visitor daily of the care providers during their stay. The number of respondents who received information about the health of their children was 164 out of 202 (i.e. 81.19\%). The respondents who received advice on the adherence of anti-malarial treatment were 125 out of 202 (i.e. 61.88\%). The proportion of respondents of children who received appropriate information on malaria prevention was $36.14 \%$ (73 out of 202). As for follow-up, 133 out of 201 children $(66.17 \%)$ had received an additional oral anti-malarial treatment prescription on discharge from the hospital to cover the seven days of treatment. The remaining children (68 or $33.83 \%$ ) did not benefit. The follow-up appointment was given to 120 out of 201 children (59.70\%). 
Clinical status after cares of children suffering of severe malaria,june-july 2017

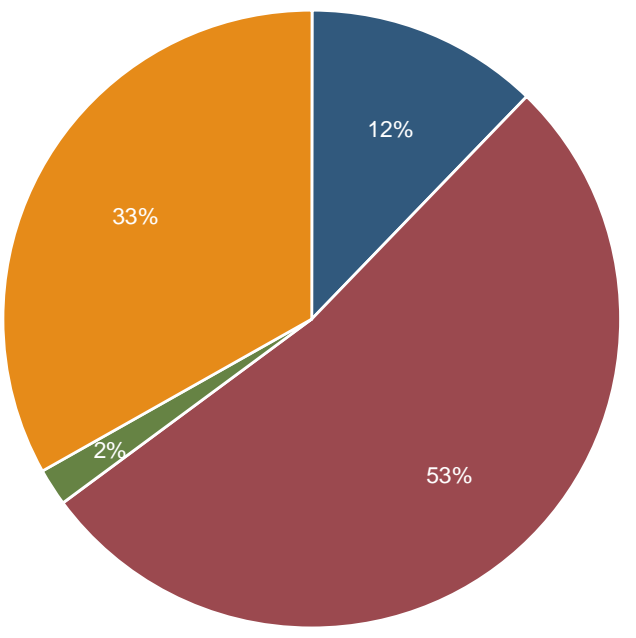

Healed (confirm by TDR or GE)

Improved clinical status (not confirm by TDRou GE)

Deceased

State not determined (patient discharged against medical advice)

Figure 2. Outcome of severe malaria case management in children under five years in four hospitals of the department of Littoral (Benin) in 2017.

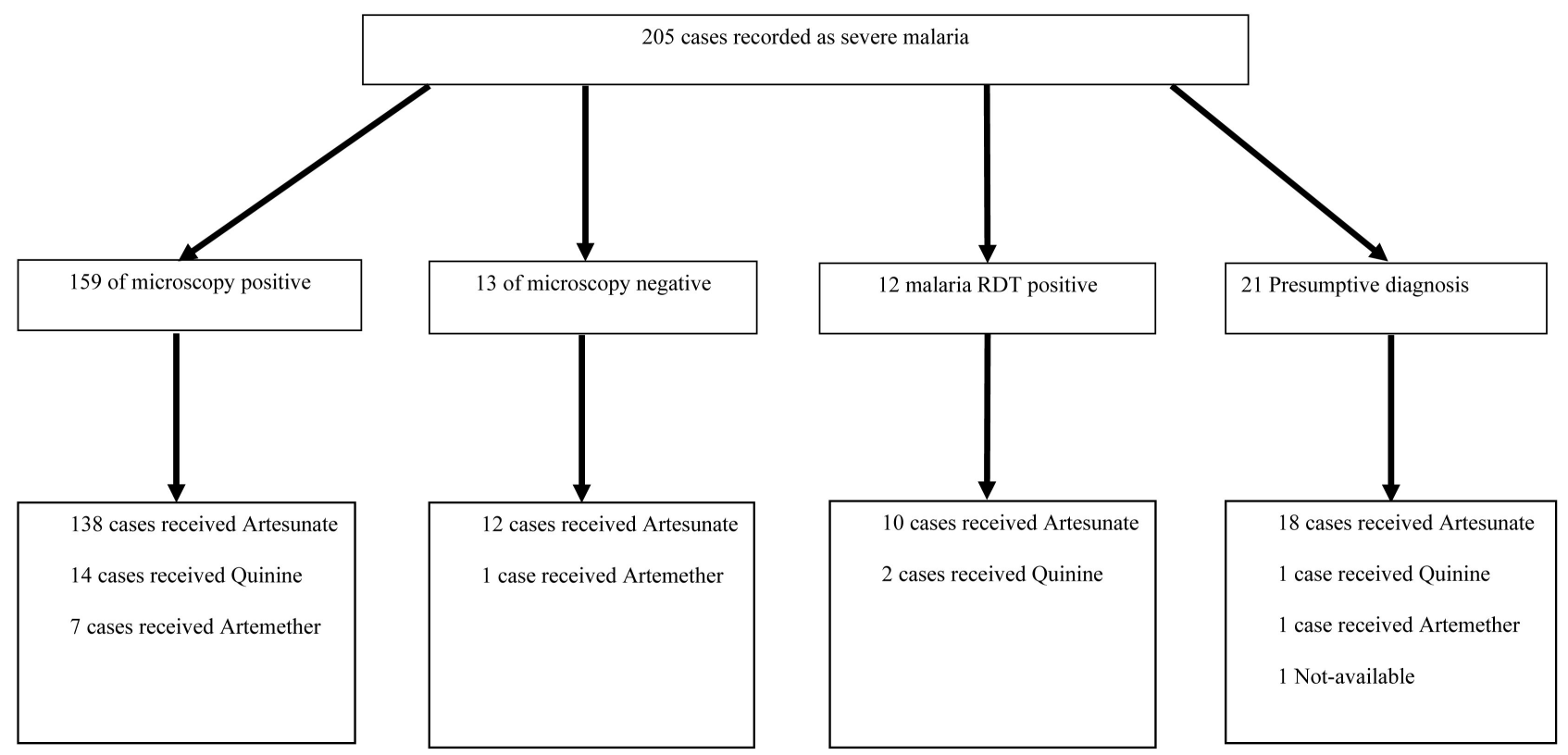

Figure 3. Diagnosis and treatment of severe malaria cases in children under five years in four hospitals of the department of Littoral (Benin).

\subsection{The Quality Level of Severe Malaria Case Management}

The proportion of severe malaria cases managed per the requirements of the $\mathrm{Na}$ tional Protocol was $23.41 \%$ (i.e. 48 out of 205 cases). Table 2 summarises the assessment of each component of quality by the requirements set out in the $\mathrm{Na}$ tional Protocol for the management of severe malaria cases. The Cronbach alpha's test statistic was equal to 0.2554 (less than 0.7 ). Therefore, the components 
Table 2. Assessment of the components of severe malaria case management in children under five at four hospitals in littoral department (Benin).

\begin{tabular}{|c|c|c|}
\hline Components assessment & Frequency $(n=205)$ & Proportion (\%) \\
\hline \multicolumn{3}{|l|}{ Diagnosis } \\
\hline Good & 175 & 85.37 \\
\hline Bad & 30 & 14.63 \\
\hline \multicolumn{3}{|l|}{ Treatment } \\
\hline Good & 115 & 56.10 \\
\hline Bad & 90 & 43.90 \\
\hline \multicolumn{3}{|l|}{ Patient Monitoring } \\
\hline Good & 202 & 98.54 \\
\hline Bad & 3 & 1.46 \\
\hline \multicolumn{3}{|l|}{ Counselling } \\
\hline Sufficient & 154 & 75.12 \\
\hline Insufficient & 51 & 24.88 \\
\hline \multicolumn{3}{|l|}{ Follow-up } \\
\hline Good & 140 & 68.29 \\
\hline Bad & 65 & 31.71 \\
\hline
\end{tabular}

are distinct from each other and so permit to measure distinct stages of severe malaria case management.

Furthermore, the quality of patient's surveillance varies significantly between private and public health facilities $(\mathrm{p}=0.027)$. Moreover, counselling and patient's follow-up components are significantly $(\mathrm{p}<0.001)$ influenced by the respondent's decision to abandon care. Discharge without medical advice is significantly $(\mathrm{p}=0.002)$ associated with the respondent's lack of knowledge of the consequences of severe malaria.

\section{Discussion}

Three-quarters of children admitted in a health facility for a severe malaria case had not been managed per the requirements set out in the national protocol. The application of the national protocol for the management remains low (23.41\%). The different components of management are not all implemented during the care of patients. The respondent's knowledge of the consequences of severe malaria and the sector (public/private) of health facility influence severe malaria case management. Diagnosis is the most sensitive component because more than ten percent of patients were treated by non-confirmed diagnostic. In fact, health care providers use malaria RDT or presumption to diagnose patients. This result corroborated the findings in Malawi and Burkina-Faso where patients are over diagnosed or and overtreated [8] [9] [10]. The studies performed in Burkina-Faso showed that $89.1 \%$ of patients were over diagnosed [8], and 242 cases 
(14.05\%) were confirmed malaria cases against 1480 (85.95\%) presumptive cases [10]. In Malawi, 31\% of patients without confirmed malaria were overtreated with an anti-malaria [9]. The routine use of Malaria RDT for the diagnosis of malaria was not recommended especially in under five children [11] [12] [13]. When the microscopy test is not available in health facility or patient's purchasing power is insufficient, RDT of malaria is a better alternative of diagnosis [14]. The diagnosis was better performed in private health facilities, compared to public facilities $(p=0.001)$. The fact is that health care providers do not all have the same level of information on the national malaria treatment guidelines would explain the disparities in applying for the national guidelines [15], although all health care providers had treated patients with the right first-line treatment.

Many studies related to the management of malaria cases focused mainly on the components of diagnosis and treatment [8] [10] [12] [15]-[23]. In this study, however, severe malaria case management assessment was conceived to go beyond diagnosis and treatment. Other aspects of care including monitoring, counselling and patient follow-up were highlighted. In severe malaria, clinical status of children can deteriorate rapidly. So, regular observation of vital signs is essential [23]. Also, the quality of patient surveillance varies significantly between private and public health facilities $(p=0.027)$. This discrepancy could be due to the factors related to the management and organisation of the health facility [5]. Indeed, the availability of the human resource (number of health care providers), and care material varies from one health facility to another.

Moreover, counselling and patient's follow-up components are significantly ( $\mathrm{p}$ $<0.001$ ) influenced by the respondent's decision to abandon care. The abandonment of care does not allow for continuing and completing the process of care. The notification of an improvement in the child's clinical status or financial reasons appears to be the main justifications expressed by the child's respondent. Also, the lack of knowledge of the consequences of severe malaria would explain the choice of giving up health care since discharge without medical advice is significantly ( $p=0.002)$ associated with respondent's lack of knowledge of the consequences of severe malaria. These facts would reveal that the management of severe malaria cases as recommended by the NMCP is subject to external forces to the health system, especially factors related to care recipients.

The prospective data collection was a strength of the study. Indeed, as soon as the patient is admitted for a presumption of severe malaria, the data relating to his/her care are recorded as the patient is receiving care. Thus, it reduces information bias due to incomplete filling of patient records and memorisation bias among child respondent. Also, this paper includes both private and public health facilities. In fact, it allows taking count of different organisations that could affect care. The main limitation of this study relates the sampling method adopted-non-probabilistic. Thus, the results of this study cannot be generalised to all the health facilities in the country. However, as the research has demonstrated, the findings provide a very insightful picture of the way that malaria 
cases are managed in the selected health facilities in Cotonou. Further, data were collected during the rainy season, which is characterised by high transmission of malaria. Similarly, the results may not represent the case management pattern for the entire year.

Inappropriate case management of severe malaria leads to overdiagnosis, therapeutic failures and additional costs of care [19]. The National Malaria Control Program could strengthen communication strategies about severe malaria complication sequels especially in children under five years. In terms of areas for future studies, a national representative data could be collected to assess the phenomenon at the national level, and especially to study the factors that may be associated with the quality of severe malaria case management in Benin Republic.

\section{Conclusion}

The different components of management are not all implemented during the care of patients. There is a need for training to health workers on implementation of severe malaria in children under 5 years protocol. Also, there is a need for investigation about factors associated with malaria protocol implementation.

\section{Recommendation}

All components recommended for malaria case management are not implemented systematically in all patients. Efforts are still needed to raise the level of protocol implementation in health facilities. More investigation must be completed to better understanding factors which influence the implementation of each component of malaria case management in health facilities.

\section{What Is Already Known?}

The studies on malaria are often focused on diagnosis or/and therapy. They are related to diagnosis methods and their efficacy assessment, anti-malaria drug's efficacy, drug resistance, vaccine use, vector control or people's knowledge and practices.

\section{What Are the New Findings?}

This study shows a holistic approach to assess severe malaria case management components. It highlights components other than diagnosis and treatment, which are most of the time neglected but are important for correct case management. A better understanding of other components of malaria case management quality could help to improve the implementation of malaria care policies.

\section{What Do the New Findings Imply?}

All components recommended for malaria case management are not implemented systematically in all patients. Efforts are still needed to raise the level of protocol implementation in health facilities. More investigation must be com- 
pleted to better understanding factors which influence the implementation of each component of malaria case management in health facilities.

\section{Author Contributions}

The authors jointly contributed to conceptualising and review the study protocol. COAB, AROK, GAV, and GES worked to conceptualising study protocol. GAV coordinated data collection and analysed the data. EMO, GES and GAV contributed to the interpretation of results. CJS, MM performed the examination of the research methodology, and the results. The contributor JA and authors GAV, GES wrote article.

\section{Acknowledgements}

We express our sincere gratitude to all contributors in particular sir J.A. We also thank the Regional Institute of Public Health (IRSP-CAQ) team for their technical support. The authors would like to appreciate the patients, their parents and health care providers who took time to participate in this study, as well as the data collection teams.

\section{Conflicts of Interest}

The authors declared that they have not competing interests.

\section{References}

[1] Ministère de la santé (2016) Annuaire des statistiques sanitaires du Bénin année 2015.

[2] Programme National de Lutte contre le Paludisme (2016) Enquête sur les indicateurs du paludisme au Bénin (MIS 2015).

[3] Bhatt, S., Weiss, D.J., Cameron, E., et al. (2015) The Effect of Malaria Control on Plasmodium falciparum in Africa between 2000 and 2015. Nature, 526, 207-211. https://doi.org/10.1038/nature15535

[4] Programme National de Lutte contre le Paludisme (2014) Plan stratégique de lutte contre le paludisme 2011-2015 révisé avec extension sur 2018. 82.

[5] Torres, S., Tougher, R.S., Palafox, B., et al. (2009) Evidence for Malaria Medicines Policy a Qualitative Assessment of the Private Sector Antimalarial Distribution Chain in Benin Co-Investigators Principal Investigator. https://researchonline.lshtm.ac.uk/id/eprint/2869440

[6] Ministère de la santé, Programme National de Lutte contre le Paludisme (2015) Directives nationales de prise en charge des cas de paludisme. Révisée.

[7] World Health Organization (2015) Guidelines for the Treatment of Malaria-3rd Edition. http://www.who.int/malaria

[8] Yaméogo, T.M., Kyelem, C.G., Ouédraogo, S.M., et al. (2011) Diagnostic et prise en charge du paludisme grave chez l'adulte: Observance des directives nationales au Burkina Faso. Bulletin de la Société de Pathologie Exotique, 104, 284-287. https://doi.org/10.1007/s13149-010-0129-x

[9] Steinhardt, L.C., Chinkhumba, J., Wolkon, A., et al. (2014) Quality of Malaria Case Management in Malawi: Results from a Nationally Representative Health Facility 
Survey. PLoS ONE, 9, e89050. https://doi.org/10.1371/journal.pone.0089050

[10] Yaméogo, T.M., Tapsoba, S.P., Sombié, I., et al. (2015) Évaluation de l'application des directives nationales de prise en charge des cas de paludisme au CHU de Bobo-Dioulasso. Sante Publique (Paris), 27, 265-273.

https://doi.org/10.3917/spub.152.0265

[11] Wilson, M.L. (2012) Malaria Rapid Diagnostic Tests. Clinical Infectious Diseases, 54, 1637-1641. https://doi.org/10.1093/cid/cis228

[12] Elechi, H.A., Rabasa, A.I., Bashir, M.F., et al. (2015) Uncomplicated Malaria in Children: The Place of Rapid Diagnostic Test. Nigerian Medical Journal, 56, 85-90. https://doi.org/10.4103/0300-1652.150686

[13] Vernelen, K., Barbé, B., Gillet, P., et al. (2018) Photo-Based External Quality Assessment of Malaria Rapid Diagnostic Tests in a Non-Endemic Setting. PLoS ONE, 13, e0201622.

[14] Rakotoarivelo, R.A., Razakarison, C., Gottot, S., et al. (2012) Évaluation de la prise en charge des cas de fièvre et du paludisme par des médecins communautaires dans les hautes terres centrales de Madagascar, 2009-2010. Médecine et Santé Tropicales, $22,317-322$

[15] Ouédraogo, L.T., Drabo, K.M. and Zongo, P.S. (2012) Évaluation de l'application du protocol national de traitement des cas de paludisme simple en milieu rural au Burkina Faso. Sante Publique (Paris), 24, 353-363. https://doi.org/10.3917/spub.124.0353

[16] Msangeni, H.A., Kamugisha, M.L., Sembuche, S.H., et al. (2011) Prospective Study on Severe Malaria among In-Patients at Bombo Regional Hospital, Tanga, North-Eastern Tanzania. BMC Infectious Diseases, 11, 256. https://doi.org/10.1186/1471-2334-11-256

[17] Djadou, K.E., Takassi, E.O., Guedenon, J.K., et al. (2018) Severe Malaria in Children at Tsevie Hospital (Togo). 2017 International Rural and Elderly Health Informatics Conference, Lome, 13-17 December 2017, 1-4. https://doi.org/10.1109/IREEHI.2017.8350468

[18] Makumbe, B., Tshuma, C., Shambira, G., et al. (2017) Evaluation of Severe Malaria Case Management in Mazowe District, Zimbabwe, 2014. The Pan African Medical Journal, 27, 33. https://doi.org/10.11604/pamj.2017.27.33.11081

[19] Elmansouf, L., Dubos, F., Dauriac, A., et al. (2011) Évaluation des pratiques dans la prise en charge du paludisme d'importation de l'enfant dans la région Nord-Pas-de-Calais. Médecine et Maladies Infectieuses, 41, 145-151.

[20] Gbadoé, A.D., Lawson-Evi, K., Badayodi, A., et al. (2006) Paludisme grave de l'enfant: Évaluation de la prise en charge des formes anémiques et neurologiques dans un service de réanimation en milieu tropical. Archives de Pédiatrie, 13, 1554-1555. https://doi.org/10.1016/j.arcped.2006.09.011

[21] Raobijaona, H., Randrianotahina, C.H. and Razanamparany, M. (2000) Le paludisme grave de l'enfant observé au Service de Pédiatrie du Centre Hospitalier de Befelatanana à Antananarivo (Madagascar) en 1996-1998. Archives de P Institut Pasteur de Madagascar, 66, 23-25.

[22] Olupot-Olupot, P. and Maitland, K. (2013) Management of Severe Malaria: Results from Recent Trials. Advances in Experimental Medicine and Biology, 764, 241-250.

[23] Crawley, J., Chu, C., Mtove, G., et al. (2010) Malaria in Children. The Lancet (London, England), 375, 1468-1481. https://doi.org/10.1016/S0140-6736(10)60447-3 


\section{List of Abbreviations}

\begin{tabular}{ll}
\hline NMCP & National Malaria Control Program \\
RDT & Rapid Diagnostic Test \\
TDR & "Test de Diagnostique Rapide" \\
IRSP-CAQ & Institut Régional de Santé Publique Comlan Alfred Quenum \\
GE & "Goutte Epaisse" \\
WHO & World Health Organisation \\
IPT-sp & Intermittent Preventive Treatment with Sulfadoxine/Pyrimethamine \\
LLINs & Long-Lasting Insecticidal Nets \\
ACTs & Artemisinin-based Combinations Therapies \\
MIS & Malaria Indicators Surveys \\
CI & Confidence Interval \\
Q1 & first Quartile \\
Q3 & third Quartile \\
\hline
\end{tabular}

